\title{
Produção e Avaliação da Qualidade de Lâminas de Madeira de um Híbrido de Pinus elliottii var. elliottii $\times$ Pinus caribaea var. hondurensis
}

\author{
Natalie Ferreira de Almeida ${ }^{1}$, Geraldo Bortoletto Jr. ${ }^{2}$, \\ Rafael Farinassi Mendes ${ }^{3}$, Paula Gabriela Surdi²
}

\author{
${ }^{1}$ Departamento de Ciências Florestais, Universidade de São Paulo - USP, Piracicaba/SP, Brasil \\ ${ }^{2}$ Departamento de Ciências Florestais - LCF, Universidade de São Paulo - USP, Piracicaba/SP, Brasil \\ ${ }^{3}$ Departamento de Ciências Florestais, Universidade Federal de Lavras - UFLA, Lavras/MG, Brasil
}

\begin{abstract}
RESUMO
Neste trabalho, objetivou-se avaliar o potencial da madeira do híbrido Pinus elliottii var. elliottii $\times$ Pinus caribaea var. hondurensis, aos sete anos e dez meses de idade, para produção de lâminas. Foram avaliados os seguintes aspectos: a densidade da madeira, o rendimento do processo de laminação e a qualidade das lâminas produzidas a partir de toras retiradas de duas posições no tronco - uma da base (A) e outra subsequente (B). A madeira apresentou baixa densidade básica $\left(0,320 \mathrm{~g} / \mathrm{cm}^{3}\right)$ e demonstrou facilidade para ser laminada. O rendimento médio do processo de laminação das toras, sem casca, foi de aproximadamente 53\%. Lâminas classificadas nas classes $\mathrm{C}$ e $\mathrm{C}+$ somadas corresponderam a aproximadamente $61 \%$ do total obtido. As toras da base (A) renderam maior número de lâminas, no entanto, de qualidade inferior em relação às produzidas pelas toras $(\mathrm{B})$. Conclui-se que a madeira do híbrido estudado apresentou bom potencial para a produção de lâminas.
\end{abstract}

Palavras-chave: lâminas, rendimento, híbrido de pinus.

\section{Production and Quality Evaluation of Veneers of Hybrid Pinus Wood}

\begin{abstract}
This work aimed to evaluate the utilization potential of wood from Pinus elliottii var. elliottii $\mathrm{x}$ Pinus caribaea var. hondurensis hybrid, seven years and 10 months of age, for veneer production. Wood density, peeling yield and the quality of the veneers produced from logs taken from two positions on the tree trunk were assessed: from the base (A logs) and subsequent (B logs). The wood presented low basic density $\left(0.320 \mathrm{~g} / \mathrm{cm}^{3}\right)$ and proved to be easily processed in the rotary cutting lathe. The peeling yield (average based on logs without bark) was approximately $53 \%$. The veneers graded in the $\mathrm{C}$ and $\mathrm{C}+$ classes accounted for approximately $61 \%$ of the total obtained. The A logs yielded more veneers, but the veneers produced presented lower quality compared with those produced from the B logs. We concluded that the hybrid wood herein studied shows good potential for veneer production.
\end{abstract}

Keywords: veneers, yield, hybrid Pinus. 


\section{INTRODUÇÃO}

Existe uma grande variedade de espécies de madeiras com características adequadas para a produção de lâminas e painéis compensados; no entanto, a grande dificuldade está em encontrar espécies que possam promover o fornecimento contínuo de madeira e que possibilitem a produção de toras de tamanhos e formas adequadas para a laminação (Walker, 1993).

As espécies de rápido crescimento, tais como pinus e eucalipto, apresentam grande potencial para a laminação e produção de compensados, em função das boas características da sua madeira, por serem espécies de rápido crescimento e também devido à disponibilidade de grandes áreas plantadas no Brasil.

Apesar de diversos autores apontarem que as espécies de rápido crescimento podem apresentar algumas limitações técnicas relacionadas à madeira - sobretudo, quanto aos aspectos de densidade, porosidade, presença de madeira de reação e grande proporção de madeira juvenil -, pesquisas sobre o comportamento da madeira destas espécies para laminação e produção de painéis compensados têm sido amplamente realizadas no Brasil; note-se que as lâminas de pinus já vêm sendo utilizadas pelas indústrias, desde o início da década de 1990 (Iwakiri et al., 2000).

A utilização da madeira de Pinus spp no Brasil encontra-se diversificada, gerando produtos não só como lâminas, painéis compensados e madeira serrada, mas também painéis reconstituídos (MDP, MDF e OSB), destinados principalmente aos setores da construção civil, de móveis e embalagens, atendendo aos mercados interno e externo. Apesar do alto grau de diversificação atual, a cada dia surgem novos produtos e aplicações resultantes do esforço contínuo de pesquisas realizadas em universidades, institutos e empresas do país e do mundo (Bortoletto Jr, 2006).

No ano de 2000, a Empresa Pinus Brasil deu início à implantação de talhões experimentais plantados com o híbrido Pinus elliottii var. elliottii (PEE) $\times$ Pinus caribaea var. hondurensis (PCH). Estudos conduzidos até o momento, pela mesma empresa, sobre o desenvolvimento do híbrido em comparação com amostras fiéis de outros plantios puros de Pinus elliottii e Pinus taeda revelaram que, aos 5,6 anos de idade, o híbrido apresentou incremento médio anual (IMA) de $44,8 \mathrm{~m}^{3} / \mathrm{ha} / \mathrm{ano}$, o Pinus elliottii, IMA de $18,8 \mathrm{~m}^{3} / \mathrm{ha} /$ ano, e o Pinus taeda, IMA de $28,5 \mathrm{~m}^{3} / \mathrm{ha} /$ ano, o que seria uma confirmação da viabilidade de investimento no híbrido (Pinus Brasil, 2009).

O híbrido em referência, sob o ponto de vista silvicultural, apresenta um alto potencial no que diz respeito à produção de madeira; porém, são escassas as referências na literatura sobre a qualidade dessa madeira e do seu potencial de aplicação tecnológico para geração de produtos madeireiros específicos, especialmente no Brasil.

Extensos plantios experimentais com o híbrido foram estabelecidos na Austrália e a espécie demonstrou superioridade em crescimento, adaptação a uma ampla variedade de sítios e retilinidade do tronco igual ou superior a ambas as espécies parentais, quando cultivado em locais com solos mal drenados (Nikles, 2000).

Neste contexto, o estudo do potencial da madeira deste híbrido de Pinus para a produção de lâminas é importante, na medida em que visa a gerar subsídios para uma futura utilização dessa madeira em escala comercial e aumentar as fontes de matéria-prima qualificada para o referido segmento da indústria madeireira. Neste trabalho, objetivou-se avaliar a densidade da madeira, o rendimento em laminação e a qualidade das lâminas produzidas de duas posições de retirada das toras no tronco (A e B) de árvores de $\mathrm{PEE} \times \mathrm{PCH}$.

\section{MATERIAL E MÉTODOS}

\subsection{Coleta do material e produção de lâminas}

As árvores do $\mathrm{PEE} \times \mathrm{PCH}$ foram cedidas pela Empresa Pinus Brasil Agro Florestal LTDA e coletadas nos plantios da Fazenda Pedra Maria, localizada na região de Buri-SP. No talhão em que se deu a seleção, não houve prática de desrama e a implantação foi realizada em fevereiro de 2002, com um espaçamento de $3 \times 3 \mathrm{~m}$, a partir de mudas provenientes de sementes.

Foram selecionadas cinco árvores, com sete anos e dez meses de idade, e diâmetro à altura do peito (DAP) de aproximadamente $30 \mathrm{~cm}$. Na seleção, foi 
dada preferência para árvores com tronco cilíndrico, reto, sem bifurcações ou outros defeitos visuais que poderiam incompatibilizar o material com o seu processamento.

As árvores selecionadas foram abatidas e, a partir da base de cada uma delas, foi retirada uma tora de 2,50 metros. As toras ficaram armazenadas em um tanque de acondicionamento com água até o seu processamento.

Em seguida, das toras de 2,50 metros de comprimento, foram amostrados, subsequentemente, a partir da base: um disco (I) com $5 \mathrm{~cm}$ de espessura; uma tora (A) de 1,10 m de comprimento; um disco (II), uma tora (B) e um disco (III). Dessa forma, no total, foram amostradas dez toras de 1,10 $\mathrm{m}$ de comprimento e 15 discos de $5 \mathrm{~cm}$ de espessura, conforme ilustrado na Figura 1. As toras foram destinadas à laminação em torno rotativo e os discos, utilizados na determinação da densidade básica da madeira de acordo com a Norma NBR 11941-02 (ABNT, 2003).

As toras, depois de descascadas, foram aquecidas em água com temperatura de aproximadamente $55^{\circ} \mathrm{C}$, por um período de 24 horas, conforme procedimento usual na indústria de laminação de Pinus, também adotado por Medina (1986) e Bortoletto Jr (2006).

As lâminas, com espessura nominal de 2,0 mm, foram obtidas através do torno rotativo Thons e Benato, modelo LHT-14, com a seguinte regulagem: a) ângulo de afiação da faca de $21^{\circ} 00^{\prime}$; b) ângulo da faca variando automaticamente de $90^{\circ} 00^{\prime}$ a $89^{\circ} 00^{\prime}$; c)

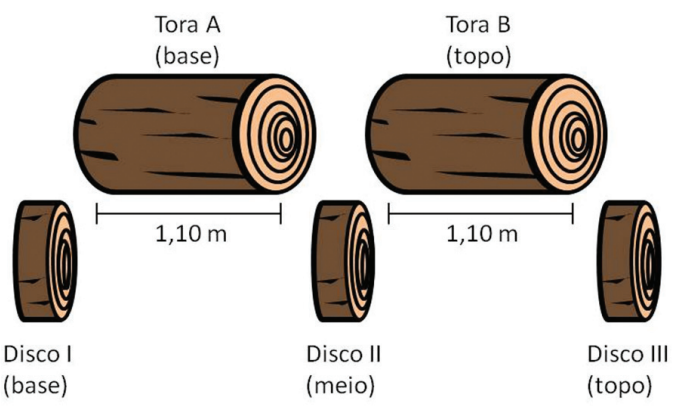

Figura 1. Esquema de retirada das toras para a laminação e dos discos para a determinação da densidade básica da madeira.

Figure 1. Scheme for the logs and disks withdrawal for peeling process and the wood basic density determination. abertura horizontal de $1,80 \mathrm{~mm}$; d) abertura vertical de $0,45 \mathrm{~mm}$.

Durante o desenrolamento das toras, as lâminas foram enroladas em bobinas e, em seguida, guilhotinadas antes do seu resfriamento. As dimensões das lâminas guilhotinadas foram de 0,002 $\times 1,00 \times 1,10$ metro.

\subsection{Determinação do rendimento em laminação}

A determinação do rendimento da laminação foi baseada nos procedimentos adotados por Medina (1986) e Bortoletto Jr (2006). Para efetuar os cálculos e proceder a tal determinação, foram realizadas medidas dos diâmetros das toras com casca e sem casca, das toras arredondadas e dos rolos-resto da laminação.

Os volumes das toras entre as diferentes operações foram calculados de acordo com as fórmulas a seguir:

Volume da tora com casca (V1) e sem casca (V2): $V=(\pi / 8) \times\left(\right.$ Dmaior $^{2}+$ Dmenor $\left.^{2}\right) \times L$

Volume da tora arredondada (V3) e do roloresto (V4):

$V=(\pi / 4) \times D^{2} \times L$

\section{Volume verde laminado (V5):}

$V=V_{3}-V_{4}$

em que: $\mathrm{D}=$ Diâmetro da tora $(\mathrm{m}) ; \mathrm{L}=$ Comprimento da tora $(\mathrm{m}) ; \mathrm{V}=$ Volume $\left(\mathrm{m}^{3}\right)$.

O rendimento do processo de laminação foi calculado de acordo com as seguintes perdas:

Perda com o descascamento (\%):

$\left(V_{1}-V_{2}\right) \times 100=V_{1}$

\section{Perda com o arredondamento (\%):}

$\left(V_{2-} V_{3}\right) \times 100=V_{2}$

\section{Perda com o rolo-resto (\%):}

$\left(V_{3-} V_{5}\right) \times 100=V_{2}$

em que: $\mathrm{V}_{1}=$ Volume com casca $\left(\mathrm{m}^{3}\right) ; \mathrm{V}_{2}=$ Volume sem casca $\left(\mathrm{m}^{3}\right) ; \mathrm{V}_{3}=$ Volume arredondado $\left(\mathrm{m}^{3}\right)$; $\mathrm{V}_{4}=$ Volume do rolo-resto $\left(\mathrm{m}^{3}\right) ; \mathrm{V}_{5}=$ Volume verde laminado $\left(\mathrm{m}^{3}\right)$. 


\subsection{Secagem das lâminas e classificação}

As lâminas, depois de guilhotinadas, foram colocadas sobre suportes de madeira para secagem natural, dispostas paralelamente ao plano horizontal, em local coberto, até atingirem a umidade de equilíbrio com o ambiente (umidade entre 10 e 12\%). Em seguida, foram classificadas, utilizando-se o método visual, segundo a presença de defeitos (nós e rachaduras), em classes de qualidade decrescentes (A, B, C+, C, D), conforme o Programa Nacional de Qualidade da Madeira - Compensado de Pinus (ABIMCI, 2002), baseado na Norma NBR 9531 (ABNT, 1996).

Para a classificação das lâminas, foi obtido o número de lâminas de cada classe e sua percentagem com relação ao número total de lâminas de cada uma das toras (A e B).

\section{RESULTADOS E DISCUSSÃO}

\subsection{Densidade básica da madeira}

Os valores médios de densidade básica e teor de umidade da madeira do híbrido de PEE $\times$ PCH encontram-se na Tabela 1.

Observa-se na Tabela 1 que a densidade básica média da madeira do híbrido de PEE $\times$ PCH foi de $0,320 \mathrm{~g} / \mathrm{cm}^{3}$.

Segundo Lutz (1978), espécies de madeira consideradas adequadas para laminação possuem densidade básica variando entre 0,40 e $0,70 \mathrm{~g} / \mathrm{cm}^{3}$, e de acordo com Walker (1993), esse valor pode variar

Tabela 1.Valores médios de densidade básica e teor de umidade da madeira do híbrido de PEE $\times$ PCH.

Table 1. Average values of basic density and moisture content of hybrid wood of the PEE $\times$ PCH.

\begin{tabular}{ccc}
$\begin{array}{c}\text { Posição do } \\
\text { Disco }\end{array}$ & $\begin{array}{c}\text { Densidade } \\
\text { Básica }^{\mathbf{1}}\left(\mathbf{g} / \mathbf{c m}^{3}\right)\end{array}$ & $\begin{array}{c}\text { Teor de umidade da } \\
\text { Madeira }^{2}(\%)\end{array}$ \\
\hline Base & $0,339 \mathrm{~b}$ & 209,1 \\
Meio & $0,313 \mathrm{a}$ & 229,1 \\
\hline Topo & $0,310 \mathrm{a}$ & 235,3 \\
\hline Média & 0,320 & 224,5 \\
\hline CV (\%) & 4,67 & 6,45 \\
\hline
\end{tabular}

Médias seguidas de mesma letra não diferem estatisticamente pelo teste de Tukey $(\alpha=0,05) .{ }^{1}$ Massa obtida a $0 \%$ de umidade e o volume da madeira totalmente saturada em água; ${ }^{2}$ Teor de umidade da madeira totalmente saturada em água. entre 0,38 e $0,70 \mathrm{~g} / \mathrm{cm}^{3}$. Observa-se que o valor médio de densidade básica da madeira do presente estudo está um pouco abaixo da faixa sugerida por ambos os autores. No entanto, de acordo com Bortoletto Jr (2003), estes valores constituem um parâmetro mais apropriado para inferir sobre a facilidade em laminar uma determinada espécie de madeira, não se traduzindo como uma restrição à laminação de madeiras de baixa densidade ou alta densidade.

A madeira do híbrido deste estudo apresenta valor de densidade básica um pouco inferior aos valores encontrados na literatura para várias espécies de Pinus, tais como Pinus merkusii $\left(0,44 \mathrm{~g} / \mathrm{cm}^{3}\right)$, aos 26 anos de idade (Bortoletto Jr, 2008); P. caribaea var. bahamensis $\left(0,35\right.$ a $\left.0,50 \mathrm{~g} / \mathrm{cm}^{3}\right) ; P$. caribaea var. caribaea $\left(0,35\right.$ a $\left.0,50 \mathrm{~g} / \mathrm{cm}^{3}\right) ; P$. caribaea var. hondurensis $\left(0,35 \mathrm{a} 0,50 \mathrm{~g} / \mathrm{cm}^{3}\right) ; P$. oocarpa $(0,45$ a 0,60 $\left.\mathrm{g} / \mathrm{cm}^{3}\right) ;$ P. patula $\left(0,38\right.$ a $\left.0,50 \mathrm{~g} / \mathrm{cm}^{3}\right)$, e P. taeda $(0,47$ a $0,51 \mathrm{~g} / \mathrm{cm}^{3}$ ) (Carpanezzi et al., 1986). No entanto, deve-se considerar que a madeira do híbrido possuía apenas sete anos e dez meses de idade, ou seja, os valores inferiores de densidade básica da madeira podem estar relacionados com a maior proporção de madeira juvenil, que geralmente ocorre em árvores jovens. Além disso, devem-se considerar o elevado crescimento e a produtividade do híbrido estudado.

Rezende et al. (2008) avaliaram a produtividade e a densidade da madeira do híbrido Pinus caribaea var. hondurensis $\times$ Pinus tecunumannii em comparação com um seminal de Pinus caribaea var. hondurensis, e concluíram que o híbrido teve produtividade em volume de até $23 \%$ maior do que o tratamento seminal; entretanto, houve diminuição de aproximadamente $10 \%$ na densidade da madeira.

Harding \& Copley (2000) fizeram um levantamento dos principais estudos realizados, desde 1971, sobre as propriedades da madeira do híbrido PEE $\times$ PCH e seus parentais; a partir dos resultados, os autores observaram que, de modo geral, a madeira do híbrido apresentou valores médios intermediários de densidade básica (405 a $508 \mathrm{~kg} / \mathrm{m}^{3}$ ) em relação aos parentais. A densidade da madeira do PEE foi superior (421 a $530 \mathrm{~kg}$ / $\mathrm{m}^{3}$ ), enquanto que a madeira do $\mathrm{PCH}$ apresentou densidade inferior (420 a $487 \mathrm{~kg} / \mathrm{m}^{3}$ ). Apesar disso, segundo os autores, as diferenças entre as densidades das madeiras do híbrido e de seus parentais foram 
muito pequenas, muitas vezes não significativas estatisticamente, isto é, de pouco significado prático para o mercado de madeiras.

Considerando-se a posição de retirada dos discos (base, meio e topo), observa-se que a densidade da madeira do híbrido do presente estudo decresceu da base para o topo, ocorrendo o contrário com o teor de umidade, cujas tendências, pode-se dizer, são condizentes com o que dispõe a literatura. A madeira da base das toras apresentou valor médio de densidade básica, estatisticamente diferente e superior ao das demais posições, as quais, por sua vez, mostraram valores equivalentes.

Para espécies do gênero Pinus, a redução da densidade com o aumento da altura de amostragem é esperada e decorre do efeito da idade, pois, a maiores alturas, há menor número de anéis de crescimento e maior proporção de lenho juvenil (Muñiz, 1993). Conforme Tsoumis (1991), quanto maior for a densidade, menor será o teor de umidade que a madeira poderá conter na saturação, devido à menor proporção relativa de espaços vazios disponíveis.

\subsection{Rendimento do processo de laminação}

A Tabela 2 apresenta os diâmetros médios das toras com e sem casca, arredondadas e dos rolosresto, obtidos das toras da base (A) e das toras do topo (B), que foram destinadas à laminação.
A Tabela 3 apresenta as perdas médias geradas pelo processo de laminação com descascamento, arredondamento, rolo-resto e a perda total, bem como o rendimento final do processo de laminação, com e sem casca, e o fator de forma das toras.

Pode-se observar através dos dados da Tabela 3 que, de modo geral, a maior perda média do processo de laminação ocorreu na etapa de arredondamento $(32,14 \%)$, seguida pelo descascamento $(23,78 \%)$ e pelo rolo-resto $(15,11 \%)$.

A perda com o arredondamento foi ligeiramente maior para as toras A (base), cujo fator de forma foi ligeiramente inferior, em relação às toras $\mathrm{B}$ (topo). Isso significa que a diferença entre as perdas pode ter sido causada pela maior conicidade das toras da base.

A perda por arredondamento encontrada por Bortoletto Jr (2008), laminando madeira de Pinus merkusii, aos 26 anos de idade, foi de $26,0 \%$ para as toras retiradas mais próximas da base da árvore e de $15,1 \%$ para as toras subsequentes. O autor justificou a diferença entre as perdas em função da conicidade mais acentuada das toras quanto mais próximas da base.

A perda média devida ao arredondamento das toras, determinada no presente estudo, foi compatível com as encontradas nos trabalhos de Medina (1986), laminando toras de Pinus elliottii aos 20 anos de idade, e de Jankowsky (1978), laminando

Tabela 2. Diâmetros médios das toras com e sem casca, arredondadas e rolos-resto.

Table 2. Average diameters of logs with and without bark, cylindrical logs and peeler core.

\begin{tabular}{|c|c|c|c|c|c|c|}
\hline \multirow{3}{*}{ Tora } & \multicolumn{6}{|c|}{ Diâmetro $(\mathrm{cm})$} \\
\hline & \multicolumn{2}{|c|}{ Maior } & \multicolumn{2}{|c|}{ Menor } & \multirow{2}{*}{ Arredondado } & \multirow{2}{*}{ Rolo-restc } \\
\hline & Com casca & Sem casca & Com casca & Sem casca & & \\
\hline $\mathrm{A}$ & 33,1 & 29,0 & 30,4 & 26,3 & 22,7 & 10,3 \\
\hline B & 29,9 & 26,1 & 27,8 & 24,4 & 20,8 & 10,2 \\
\hline
\end{tabular}

Tabela 3. Perdas geradas nas diferentes etapas da laminação, rendimento final do processo e fator de forma médio das toras.

Table 3. Losses generated in the different peeling stages, peeling yield and average of the taper.

\begin{tabular}{|c|c|c|c|c|c|c|c|}
\hline \multirow{2}{*}{ Tora } & \multicolumn{4}{|c|}{ Perdas (\%) } & \multicolumn{2}{|c|}{ Rendimento (\%) } & \multirow{2}{*}{$\begin{array}{c}\text { Fator de } \\
\text { Forma }\end{array}$} \\
\hline & Desc. ${ }^{*}$ & Arred. ${ }^{\star *}$ & Rolo-resto & Total & Com casca & Sem casca & \\
\hline A & 24,11 & 32,32 & 13,84 & 70,27 & 29,73 & 53,83 & 0,92 \\
\hline B & 23,46 & 31,96 & 16,39 & 71,81 & 28,19 & 51,66 & 0,93 \\
\hline Média & 23,78 & 32,14 & 15,11 & 71,04 & 28,96 & 52,74 & 0,925 \\
\hline
\end{tabular}

${ }^{\star}$ Desc. $=$ Descascamento ${ }^{* *}$ Arred. $=$ Arredondamento 
toras de Pinus strobus aos 12 anos de idade, cujas perdas foram de $29,9 \%$ e $32,0 \%$, respectivamente; tais valores revelam que o fator de forma das toras empregadas nesses estudos teriam sido semelhantes.

Observa-se, na Tabela 3, que a perda média geral devida ao descascamento $(23,78 \%)$ pode ser considerada relativamente alta, quando comparada com certas espécies de Pinus, como, por exemplos, Pinus elliottii (9,18\%), encontrado por Medina (1986), e Pinus merkusii (13,6\%), encontrado por Bortoletto Jr (2008). Também revela que o híbrido em estudo, na idade em que foi coletado, apresenta um teor de casca relativamente elevado. A perda média com o descascamento das toras A apresentou um valor médio ligeiramente maior em relação às toras $\mathrm{B}$, indicando que o teor de casca presente nas partes mais próximas da base da árvore foi ligeiramente maior.

Conforme Tsoumis (1991), a participação da casca no volume total de uma árvore pode ser consideravelmente diferente, dependendo da espécie, da idade, das dimensões e de outros fatores. Couto \& Brito (1982), através de um levantamento realizado em povoamentos de Pinus caribaea var. hondurensis plantados no Recôncavo Baiano, observaram que o teor de casca tendeu a reduzir-se com o aumento da idade de cinco para oito anos, sendo que o percentual de casca médio das árvores foi de 29,49\% e 27,37\%, respectivamente. $\mathrm{O}$ teor de casca encontrado por esses autores para a referida espécie, especialmente na idade de oito anos, é compatível com o teor de casca do híbrido do presente estudo.

Conforme visto anteriormente, a perda média geral com o rolo-resto $(15,11 \%)$ foi a menor dentre as demais. As toras B apresentaram perda média com o rolo-resto (16,39\%) pouco superior em relação às toras $\mathrm{A}(13,84 \%)$. Este fato deve-se ao menor diâmetro médio inicial das toras $\mathrm{B}$ em relação às toras da base $(\mathrm{A})$, uma vez que o diâmetro médio dos rolos-resto das toras das duas posições foi praticamente o mesmo, ou seja, 10,2 e 10,3 cm, respectivamente.

Medina (1986) encontrou uma perda média de 18,5\% com o rolo-resto laminando toras de Pinus elliottii, e Jankowsky (1978), avaliando a madeira de Pinus strobus para laminação, encontrou uma perda média de 13,6\% com o rolo-resto. Nota-se que a perda geral média com o rolo-resto $(15,11 \%)$ obtida no presente estudo foi intermediária em relação às obtidas no estudo desses dois autores.

O rendimento geral médio do processo de laminação das toras do híbrido, sem casca, foi de $52,74 \%$ e pode ser considerado bom. Esse valor é compatível com os rendimentos de laminação obtidos por Chong (1977) e Jankowsky (1978), os quais foram, respectivamente, 55,5\% para madeira de Pinus elliottii e 54,4\% para madeira de Pinus strobus, ambos baseados no volume inicial das toras sem casca.

Considerando-se o volume inicial das toras com casca (cujo teor foi elevado, conforme visto anteriormente), o rendimento geral médio do processo de laminação do híbrido foi de 28,96\% e pode ser considerado relativamente baixo. Na medida em que a casca obviamente não pode ser convertida em lâminas de madeira, o baixo rendimento de laminação da madeira do híbrido calculado com a casca inclusa é de importância secundária.

Observa-se que o rendimento final do processo de laminação, considerando-se as toras com casca e sem casca, foi ligeiramente maior para as toras A (base). Mesmo que essas toras tenham apresentado maiores perdas nos processos de descascamento e arredondamento, as perdas com rolo-resto foram menores.

\subsection{Classificação das lâminas}

Os resultados da classificação das lâminas obtidas podem ser observados na Tabela 4 . Os valores estão expressos em números de lâminas com os respectivos valores em percentagem, entre parênteses.

De acordo com os dados da Tabela 4, notase que as toras A geraram maior porcentagem de lâminas na classe de qualidade D (49,30\%) e, em seguida, nas classes de qualidade C $(32,39 \%)$ e C+ $(18,31 \%)$; já as toras $\mathrm{B}$ apresentaram maior número de lâminas na classe C $(54,24 \%)$ e, em seguida, nas classes D $(27,12 \%)$ e $\mathrm{C}+(18,64 \%)$. Esses resultados demonstram que, apesar de as toras do topo (B) terem apresentado um rendimento de laminação ligeiramente inferior, as lâminas geradas foram de melhor qualidade em relação às obtidas a partir das toras da base $(\mathrm{A})$. 
Tabela 4. Classificação de lâminas geradas a partir de duas posições da tora (A e B) da madeira do híbrido de PEE $\times \mathrm{PCH}$.

Table 4. Grading veneers from two positions of the log (A and B) of PEE x PCH hybrid wood.

\begin{tabular}{cccc} 
Classes & Lâminas da Tora A & Lâminas da Tora B & $\begin{array}{c}\text { Total de Lâminas } \\
\text { Tora A + Tora B }\end{array}$ \\
\hline A & - & - & - \\
B & - & - & - \\
C + & $13(18,31 \%)$ & $11(18,64 \%)$ & $24(18,46 \%)$ \\
C & $23(32,39 \%)$ & $32(54,24 \%)$ & $55(42,31 \%)$ \\
D & $35(49,30 \%)$ & $16(27,12 \%)$ & $51(39,23 \%)$ \\
Total & $\mathbf{7 1 ( 1 0 0 \% )}$ & $\mathbf{5 9 ( 1 0 0 \% )}$ & $\mathbf{1 3 0}(\mathbf{1 0 0 \% )}$ \\
\hline
\end{tabular}

Considerando-se o total de lâminas geradas pelas toras A e B, observa-se que houve maior proporção de lâminas classificadas na classe C $(42,31 \%)$. Os percentuais totais de lâminas das classes $\mathrm{C}$ e C+ somados perfizeram $60,77 \%$. Esse resultado pode ser considerado razoável, pois as lâminas foram obtidas de toras provenientes de árvores não desramadas. Caso a operação de desrama tivesse sido efetuada, a presença de nós teria sido reduzida e isso, certamente, minimizaria o percentual de lâminas da classe $\mathrm{D}$ e aumentaria o rendimento em lâminas das classes de melhor qualidade.

Medina (1986), utilizando a norma americana PS1-74, classificou lâminas de Pinus elliottii, obtidas de árvores não desramadas, e encontrou maior concentração de lâminas na classe $\mathrm{C}$, representando $61 \%$ do total avaliado.

\section{CONCLUSÕES}

A madeira do híbrido Pinus elliottii var. elliottii $\times$ Pinus caribaea var. hondurensis apresentou baixo valor médio de densidade básica $\left(0,320 \mathrm{~g} / \mathrm{cm}^{3}\right)$ e demonstrou facilidade para ser laminada no torno rotativo.

O rendimento médio do processo de laminação das toras (A e B) do híbrido, sem casca, foi de aproximadamente $53 \%$, e as lâminas das classes C e $\mathrm{C}^{+}$somadas perfizeram aproximadamente $61 \%$ do total obtido.

As toras da base (A) renderam maior número de lâminas; no entanto, de qualidade inferior em relação às produzidas pelas toras $B$.
O uso de tratamentos silviculturais, como a desrama, pode diminuir a presença de nós e melhorar a qualidade das lâminas.

De modo geral, conclui-se que a madeira do híbrido apresentou bom potencial para a produção de lâminas.

\section{STATUS DA SUBMISSÃO}

Recebido: 04 dez., 2012

Aceito: 18 mar., 2014

Publicado: 30 jun., 2014

\section{AUTOR(ES) PARA CORRESPONDÊNCIA}

\section{Natalie Ferreira de Almeida}

Departamento de Ciências Florestais, Universidade de São Paulo - USP, CEP 13418900, Piracicaba, SP, Brasil e-mail: natalie_amd@yahoo.com.br

\section{APOIO FINANCEIRO}

Houve suporte financeiro por meio de Bolsa de Mestrado concedida pela Coordenação de Aperfeiçoamento de Pessoal de Nível Superior (CAPES).

\section{REFERÊNCIAS}

Associação Brasileira da Indústria de Madeira Processada Mecanicamente - ABIMCI. Programa Nacional de Qualidade da Madeira. Curitiba; 2002. 20 p. Catálogo Técnico, n. 1. 
Associação Brasileira de Normas Técnicas - ABNT. NBR 11941: madeira - determinação da densidade básica. Rio de Janeiro: ABNT; 2003. 6 p.

Associação Brasileira de Normas Técnicas - ABNT. NBR 9531: chapas de madeira compensada. Rio de Janeiro: ABNT; 1996. 3 p.

Bortoletto Jr G. Avaliação da qualidade da madeira de Pinus merkusii para produção de lâminas. Scientia Forestalis 2008; 36(78): 95-103.

Bortoletto Jr G. Produção de compensados com 11 espécies do gênero Eucalyptus, avaliação das suas propriedades físico-mecânicas e indicações para utilização. Scientia Forestalis 2003; 63: 65-78.

Bortoletto Jr G. Produção de lâminas, compensado e laminated veneer limber - LVL a partir de madeira de Pinus merkusi [tese]. Piracicaba: Universidade de São Paulo; 2006.

Carpanezzi AA, Ferreira CA, Rotta E, Namikawa IS, Sturion JA, Pereira JCD, et al. Zoneamento ecológico para plantios florestais no estado do Paraná. Brasília: Embrapa, CNPF. 1986. 89 p. Documentos, n. 17.

Chong SL. Veneer yields of New Zealand: grow slash pine. New Zealand Journal of Forestry Science 1977; 7(3): 420-424.

Couto HTZ, Brito JO. Produtividade de plantações no nordeste brasileiro. Série Técnica IPEF 1982; 3(10): 7183.

Harding KJ, Copley TR. Wood property variation in Queensland-Grown Slash x Caribbean Pine Hybrids. In: Proceedings of Symposium on Hybrid Breeding and Genetics; 2000, Noosa Lakes Resort. Noosa: Queensland Forestry Research Institute; 2000.7 p.

Iwakiri S, Nielsen IR, Alberti RAR. Avaliação da influência de diferentes composições de lâminas em compensados estruturais de Pinus elliottii e Eucalyptus saligna. Cerne 2000; 6(2): 19-24.

Jankowsky IP. Qualidade das lâminas de Pinus strobus (MARTINEZ) var. chiapensis obtidas por desenrolamento. Circular Técnica IPEF 1978; 16: 50-59.

Lutz JF. Wood veneer log selection, cutting and drying. Washington: USDA; 1978. 740 p. Technical Bulletin, 1577.

Medina JC. Efeito da temperatura de aquecimento na produção de lâminas por desenrolamento e sobre a qualidade da colagem de compensados fenólicos de Pinus elliottii Engelm [tese]. Curitiba: Universidade Federal do Paraná; 1986.

Muñiz GIB. Caracterização e desenvolvimento de modelos para estimar as propriedades e o comportamento na secagem da madeira de Pinus elliottii Engelm e Pinus taeda L [dissertação]. Curitiba: Universidade Federal do Paraná; 1993.

Nikles DG. Experience with some Pinus hybrids in Queensland, Australia. In: Proceedings of Symposium on Hybrid Breeding and Genetics; 2000, Noosa. Noosa: Queensland Forestry Research Institute; 2000. p. 27-43.

Pinus Brasil Agro Florestal LTDA. [cited 2009 Aug 20]. Available from: www.pinusbrasil.com.br.

Rezende MA, Aroni AS, Costa VE, Severo ETD, Latorraca JVF. Densidade e produtividade da madeira de híbrido e seminal de Pinus caribaea. Floresta $e$ Ambiente 2008; 15(2): 8-17.

Tsoumis G. Science and technology of wood: structure, properties, utilization. New York: Chapman \& Hall; 1991. 494 p.

Walker JCF. Primary wood processing: principles and practice. London: Chapman \& Hall; 1993. 595 p. 